\title{
MicroRNA-181a sensitizes human malignant glioma U87MG cells to radiation by targeting Bcl-2
}

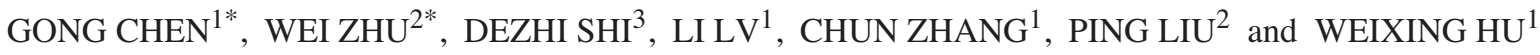 \\ Departments of ${ }^{1}$ Neurosurgery and ${ }^{2}$ Oncology, The First Affiliated Hospital \\ of Nanjing Medical University, 300 Guangzhou Road; ${ }^{3}$ Department of Neurosurgery, \\ Traditional Chinese Medicine Hospital of Jiangsu Province, Nanjing 210029, P.R. China
}

Received October 13, 2009; Accepted November 24, 2009

DOI: $10.3892 /$ or_00000725

\begin{abstract}
Radiotherapy is widely used in cancer treatment and biological studies. Multiple mechanisms induced by radiation, especially changes of the expression profile of genes, lead to the disruption of cellular homeostasis. MicroRNAs (miRNAs) are important post-transcriptional gene regulators and play an important role in response to cellular stress. Here we investigated the profiles of miRNA expression following exposure to radiation and the possible role of miRNAs in the modulation of radiosensitivity in the glioblastoma multiform U87MG cell line. MiRNA expression profiles revealed a limited set of miRNAs with altered expression in U87MG cells in response to radiation treatment. MiR-181a, a member of miR-181 family, was one of the down-regulated miRNAs, whose expression was further validated by qRT-PCR. The target mRNAs of radiation-responsive miRNAs were predicted with a target prediction tool. Transiently overexpressed miR-181a significantly sensitized malignant glioma cells to radiation treatment concurrent with the downregulation of the protein Bcl-2 (B cell lymphoma/lewkmia-2). It indicates that miR-181a may modulate radiosensitivity by targeting Bcl-2 in human malignant glioma cells. These data suggest that radiation can affect miRNA expression, which regulates the cellular response, and miR-181a could be a target for enhancing the effect of radiation treatment on malignant glioma cells.
\end{abstract}

\section{Introduction}

Glioblastoma multiform (GBM), a poorly differentiated brain tumor, is typically refractory to conventional treatment.

Correspondence to: Dr Weixing Hu, Department of Neurosurgery, or Dr Ping Liu, Department of Oncology, The First Affiliated Hospital of Nanjing Medical University, 300 Guangzhou Road, Nanjing 210029, P.R. China

E-mail: weixinghu@njmu.edu.cn

E-mail: liu-ping@csco.org.cn

${ }^{*}$ Contributed equally

Key words: microRNA, miR-181a, malignant glioma, radioresistance, B cell lymphoma/lewkmia-2
Radiotherapy, with or without surgery, is the cornerstone of the treatment plan for this tumor. However, the radiotherapeutic efficiency is often limited by the occurrence of radioresistance, reflected as a diminished susceptibility of the irradiated cells to undergo apoptosis (1). As a result, this therapeutic strategy can not substantially improve the survival rate .

With the understanding of the molecular biology of brain tumors, it has been recognized that development of radioresistance is related to changes of tumor environment and the dysregulation of certain genes, including some genes involved in a variety of cell signaling pathways as well as oncogenes and tumor suppressor genes.

MicroRNAs (miRNAs) are short non-coding RNA molecules that post-transcriptionally regulate gene expression. MiRNAs bind to the 3' UTR of an mRNA and either represses its translation or result in the degradation of the target mRNA (2). Only a few studies have been performed to determine the roles of miRNAs in radiation response. MiR-34 is transcriptionally activated by P53 in response to DNA damage, directly down-regulates the mRNA targets involved in cell cycle progression (3). Exposure to $2.5 \mathrm{~Gy}$ of X-rays leads to a significant increase of miR-34a in the spleen tissue of mice (4). Caenorhabditis elegans with loss-of-function mutations in the miR-34 gene have an abnormal cellular survival response to radiation and in vitro in breast cancer cells exogenous addition of miR-34 alters cell survival post-radiation (5). Let-7 family of miRNAs are down-regulated except let-7g which is up-regulated in response to radiation treatment and activates cell survival pathways for radioresistance such as the Ras pathway in lung cancer cells (6). Other studies also demonstrate some miRNA targeting genes involved in radio-responsive mechanisms such as miR-521 (7), miR-579, 608, 548-3p and 585 (8).

Ionizing radiation activates various survival and death signaling pathways. Radiation therapy works by triggering apoptosis in cancer cells (9). In this study, we investigated the miRNA profiles of U87MG and U87MG post-radiation and found miR-181a was one of the down-regulated miRNAs after irradiation. Target prediction for miR-181a suggests that Bcl-2 is one of its targets. There is ample evidence that Bcl-2 overexpression has an association with the resistance to conventional radiation and chemotherapeutic agents (10-15). However, the role of miRNAs and how they integrate into the 
radiation signaling pathways remains largely unknown. In our study a limited set of miRNAs were differentially expressed in malignant glioma cell line U87MG in response to radiation treatment. MiR-181a, one of the down-regulated miRNAs, was demonstrated playing an important role in the radiosensitivity of malignant glioma cells by targeting Bcl-2.

\section{Materials and methods}

Cell culture. The GBM cell line U87MG was routinely cultured in EMEM medium (Invitrogen, Carlsbad, CA, USA) supplemented with $2 \%(\mathrm{w} / \mathrm{v})$ L-glutamine, $10 \%$ (v/v) fetal calf serum (Gibco BRL, Grand Island, NY), $100 \mathrm{U} / \mathrm{ml}$ penicillin and $100 \mu \mathrm{g} / \mathrm{ml}$ streptomycin in a humidified atmosphere containing $5 \% \mathrm{CO}_{2}$ at $37^{\circ} \mathrm{C}$.

Radiosensitivity assay. At room temperature, exponentially growing cells were exposed to X-rays with $0,0.5,1.0,5.0$, $10.0,15.0,20.0,25.0,30.0$ and 35.0 Gy with a $6 \mathrm{MV}$ photon beam at a dose rate of $0.4 \mathrm{~Gy} / \mathrm{min}$ using an irradiation apparatus (2100C/D WARIAN, USA). Monolayer cells in plates were placed $100 \mathrm{~cm}$ from the source. Cells were seeded in 96-well plates at a density of 2,000 cells/well, followed by irradiation on day 2 . On day 6 , radiation sensitivity of U87MG cells was assessed using 3-(4,5-dimethylthiazol2-yl)-2,5-diphenyl-tetrazolium bromide (MTT) assay. The absorbance at $490 \mathrm{~nm}$ of each well was read on a microplate spectrophotometer (Bio-Rad, Hercules, CA, USA). The radiation dose producing $50 \%$ inhibition of cell growth was estimated by the relative survival curve.

Irradiation planning and delivery. External beam irradiation was delivered on the linear accelerator with a $6 \mathrm{MV}$ photon beam. A 40x40 cm field size was utilized and the dishes with monolayer cells were placed $100 \mathrm{~cm}$ from the source. The radiation was given with conventional fractionation ( $2 \mathrm{~Gy} /$ day, $5 \mathrm{~d} / \mathrm{wk}$ ) in one direction as the treatment given to a planning target volume of patients with malignant glioma (16). Cells were irradiated to the appropriate radiation dose, which produced 50\% inhibition of growth in U87MG cells.

MiRNA microarray analysis. The irradiated cells were then maintained for $4 \mathrm{~h}$ before extracting total RNA with TRIzol ${ }^{\circledR}$ reagent (Invitrogen) according to the manufacturer's protocol. The concentration of total RNA was quantified by measuring the absorbance at $260 \mathrm{~nm}$. MiRNA fraction was further purified using a mirVana ${ }^{\mathrm{TM}}$ miRNA isolation kit (Ambion, Austin, TX). The isolated miRNAs from U87MG and U87MG post-radiation were then labeled with Hy3 using the miRCURY ${ }^{\mathrm{Tm}}$ Array Labelling kit (Exiqon, Vedbaek, Denmark) and hybridized respectively on a miRCURY ${ }^{\mathrm{TM}}$ LNA microRNA Array (V 8.1, Exiqon) after the concentration of labeled samples (17). The Genepix 4000B scanner (Axon Instruments, Union City, CA) was used to acquire the microarray images at the $635 \mathrm{~nm}$ laser. Data were analyzed with Genepix Pro 6.0 (Axon Instruments).

Quantitative real-time PCR ( $q R T$-PCR) analysis for miRNA. Cell irradiation and RNA preparation were as described above. The concentration and purity of the RNA samples were determined spectroscopically. Expression of mature miRNA was assayed using stem-loop reverse transcription followed by real-time PCR analysis (18). cDNA was synthesized according to the protocol of the reverse transcription system (Promega, Madison, WI, USA). The SYBR and U6 gene were used for detecting gene amplification and normalizing each sample, respectively. The primers used for stem-loop qRT-PCR for miR-181a and U6 snRNA are listed in Table II. The real-time PCR reactions were performed on a 7500 Fast System Real-Time PCR cycler (Applied Biosystems, Foster City, CA), according to the manufacturer's instructions. The fold-change of each miRNA from U87MG post-radiation cells relative to U87MG was calculated using the $2^{-\Delta \Delta C t}$ Method (19), where $\Delta \Delta \mathrm{Ct}=\Delta \mathrm{Ct}$ U87MG 4 h post-radiation $\Delta \mathrm{Ct}$ U87MG and $\Delta \mathrm{Ct}=\mathrm{Ct}$ miRNA-Ct U6 snRNA.

Transient transfection and Western blot analysis. Cells were plated in 6 -well plates $\left(8 \times 10^{5}\right.$ cells/well) and transfected with $100 \mathrm{nM}$ of miR-181a mimic or $100 \mathrm{nM}$ control mimic using Lipofectamine 2000 (Invitrogen, Long Island, NY, USA) according to the manufacturer's protocol after $16 \mathrm{~h}$ incubation. The miR-181a mimic and control miRNA mimic were chemically synthesized by Shanghai GenePharma Company (Shanghai, China). Cells were fed fresh medium $6 \mathrm{~h}$ post-transfection. At 24, 48 and $72 \mathrm{~h}$ respectively, cells were harvested and homogenized with lysis buffer. Total protein was separated by denaturing $12 \%$ SDS-polyacrylamide gel electrophoresis. Western blot analysis was performed as described (20). The primary antibodies for Bcl-2 and B-actin were purchased from Santa Cruz Biotechnology. Protein levels were normalized to $\beta$-actin. Fold changes were determined.

Cell proliferation assay. Cells were plated at $80 \%$ confluence at the time of transfection. The miR-181a mimic $(100 \mathrm{nM})$ or control mimic $(100 \mathrm{nM})$ were transfected using Lipofectamine 2000 in serum-free medium for 6 h. Twenty-four hours later, cells were trypsinized and seeded into 96-well culture plates at a density of 2000 cells/well in growth medium supplemented with $10 \%$ serum, which were divided into two groups. After the cells were attached, one group without irradiation, the other one was irradiated with the dose producing $50 \%$ inhibition of cell growth. Then both were cultured in a humidified chamber at $37^{\circ} \mathrm{C}$. Each day for seven consecutive days, viable cells were identified using the MTT assay. MTT $(20 \mu 1)(5 \mathrm{mg} / \mathrm{ml})$ was added to each well, followed by incubation for $4 \mathrm{~h}$ at $37^{\circ} \mathrm{C}$. The supernatants were aspirated and the reaction was terminated by adding $100 \mu 1$ DMSO. The contents of the plates were mixed for $10 \mathrm{~min}$ and the absorbance was read at $490 \mathrm{~nm}$.

Soft agar colony formation assay. Anchorage-independent growth was assessed by monitoring colony formation in soft agar. First, $0.6 \%$ agarose in growth medium was added to a 6-well plate and allowed to solidify. Then, 500 cells transfected with miR-181a mimic or control mimic per well were plated in triplicate in $0.3 \%$ agarose onto the bottom agarose. One hour later, cells were exposed to $0,2,5 \mathrm{~Gy}$ radiation. The cells were incubated at $37^{\circ} \mathrm{C}$ in a $5 \% \mathrm{CO}_{2}$ atmosphere for 3 weeks. Fresh growth medium ( $1 \mathrm{ml} /$ well) was added after 1 and 2 weeks of 
Table I. MiRNAs changed >2-fold after irradiation.

\begin{tabular}{lccc}
\hline MiRNA increased & Fold change & MiRNA decreased & Fold change \\
\hline hsa-miR-22 & 4.27 & hsa-miR-181a & 2.68 \\
hsa-miR-500 & 4.67 & hsa-miR-302b* & 2.99 \\
hsa-miR-601 & 3.21 & hsa-miR-206 & 2.33 \\
hsa-miR-379 & 2.82 & hsa-miR-582 & 2.11 \\
hsa-miR-641 & 2.53 & hsa-miR-518b & 2.04 \\
hsa-miR-548d & 2.43 & hsa-miR-107 & 2.01 \\
hsa-miR-191 & 2.38 & hsa-miR-521 & 2.37 \\
hsa-miR-335 & 2.36 & & 2.11 \\
hsa-let-7g & 7.87 & & \\
hsa-miR-646 & 2.07 & & \\
hsa-miR-22_MM1 & 3.10 & & \\
hsa-miR-9*_MM2 & 3.03 & & \\
hsa-miR-451 & 2.34 & & \\
\hline
\end{tabular}

Significance was analyzed using Student's t-test. All values have a significance of $\mathrm{P}<0.05$. miR* is the complementary strand of the corresponding miR.

incubation. Only colonies containing $>50$ cells were counted under a microscope.

Statistical analysis. Each experiment was repeated at least 3 times. The difference between means was analyzed with the Student's t-test. All statistical analyses were performed using SPSS11.0 software (Chicago, IL). Differences were considered significant at $\mathrm{P}<0.05$.

\section{Results}

Radiosensitivity. In vitro experiments revealed the radiosensitivity of U87MG cells. According to the relative survival curve (Fig. 1A) and the fitting curve analysis (Fig. 1B), radiation dose of 18.8 Gy produced $50 \%$ inhibition of growth in U87MG cells.

MiRNA expression profiles. To monitor the expression profiles of miRNAs following radiation treatment, we employed a microarray covering $96 \%$ human miRNAs of Sanger miRBase V 9.1. The radiation dose of $18.8 \mathrm{~Gy}$, which produced $50 \%$ inhibition of growth was delivered to U87MG cells according to the plan described. The concentration of total RNA extraction was $1217.99 \mathrm{ng} / \mu 1$ (radiation-treated U87MG cells) and $1972.88 \mathrm{ng} / \mu 1$ (control U87MG cells) with the value of $\mathrm{OD}_{260} / \mathrm{OD}_{280} 1.97$ and 1.98 respectively. MiRNA microarray identified 21 miRNAs as differentiallyexpressed: 13 miRNAs up-regulated (defined as a $\geq 2.0$-fold increase) and 8 down-regulated (defined as a $\geq 2.0$-fold decrease) (Table I).

Quantitative real-time PCR assay. Among the differentiallyexpressed miRNAs, miR-181a was down-regulated 2.68-fold in response to radiation treatment. The irradiation process was repeated and the RNA was extracted for the qRT-PCR. Although the expression of several altered miRNAs showed
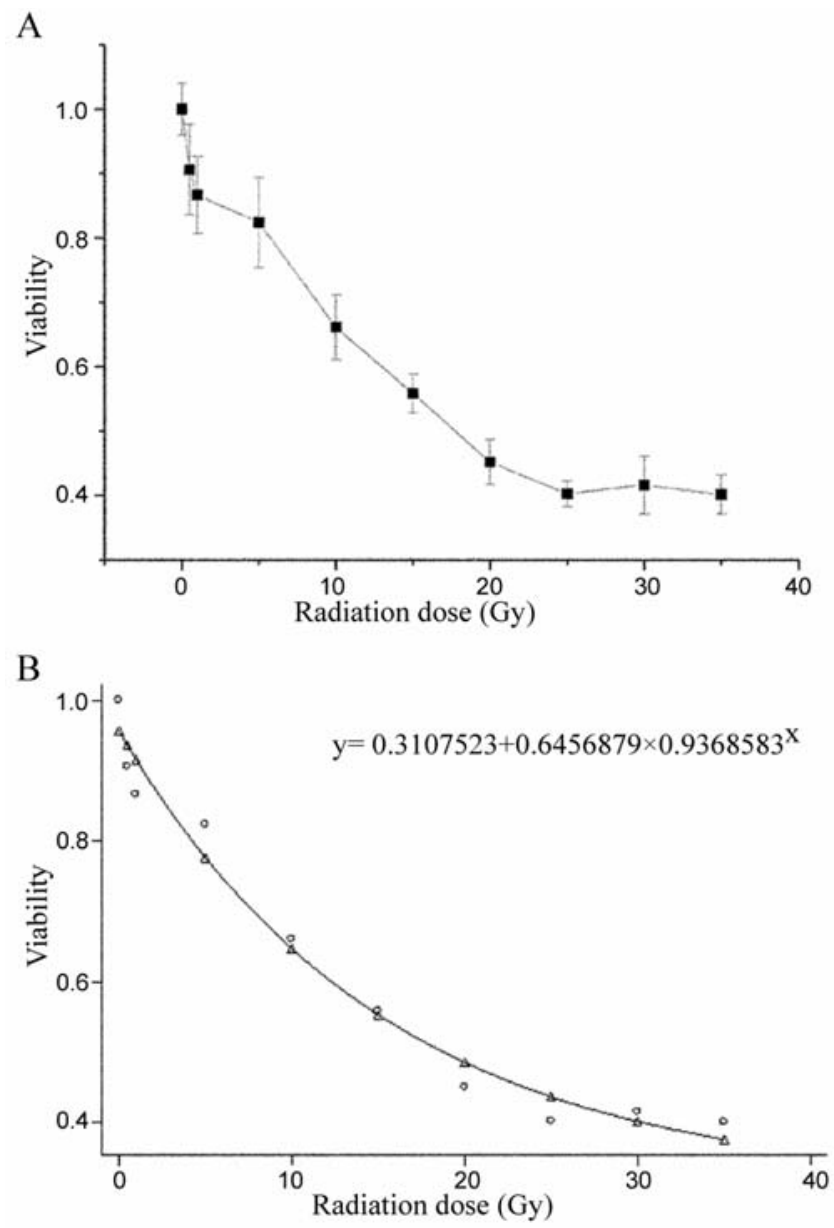

Figure 1. In vitro sensitivity of U87MG cells to radiation. Cells were seeded in 96-well plates at a density of 2,000 cells/well, followed by irradiation on day 2 . Four days later, sensitivity was assessed by MTT assay. (A) Exponentially growing cells were exposed to X-rays with $0,0.5,1.0,5.0,10.0,15.0,20.0$, 25.0, 30.0 and $35.0 \mathrm{~Gy}$. Results were expressed as a growth ratio compared with the control. (B) Curve fitting analysis indicated that radiation level of 18.8 Gy inhibits $50 \%$ cell growth in U87MG cells. (Cell growth inhibition rate $\left.\mathrm{y}=0.3107523+0.6456879 \times 0.9368583^{\mathrm{x}}, \mathrm{R}^{2}=0.9779, \mathrm{P}<0.05\right)$. 
Table II. Quantitative real-time PCR primers for U6 snRNA and miR-181a.

\begin{tabular}{lll} 
Gene & & \multicolumn{2}{c}{ Sequence } \\
\hline U6 snRNA & F primer & 5'-ATTGGAACGATACAGAGAAGAT-3' \\
& R primer & 5'-GGAACGCTTCACGAATTT-3' \\
Hsa-miR-181a & F primer & 5'-GCUGUCGGUGAGCTCAACTGGTGTCGTGGAGTCGGCAATTCAGTTGAGACTCACCG-3' \\
& R primer & 5'-ACACTCCAGCTGGGAACATTCAACGCTGTCG-3' \\
\hline
\end{tabular}

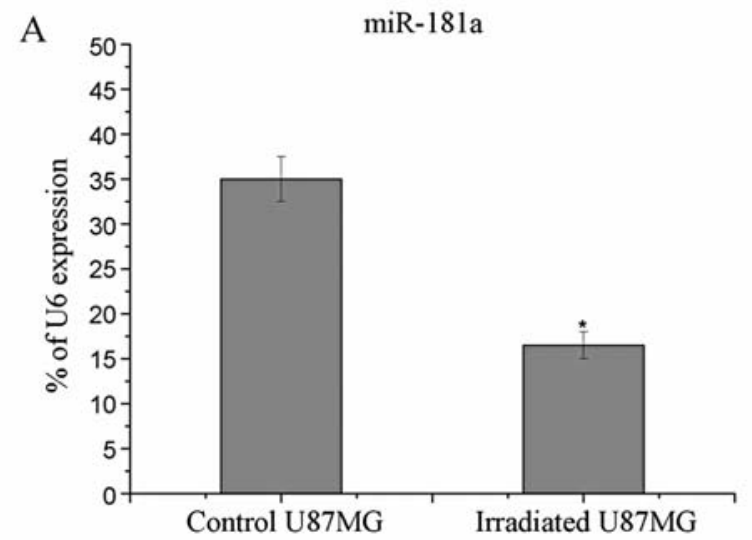

B

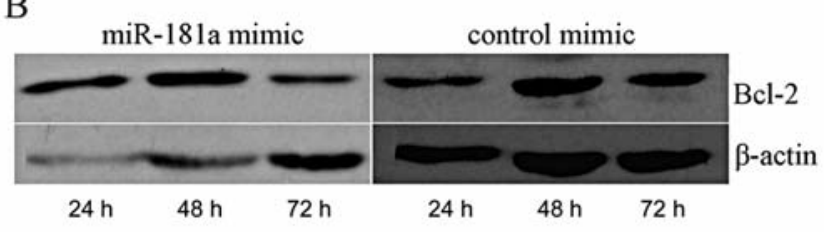

$\mathrm{C}$



Figure 2. Identification of the radiation responsive miR-181a and its target protein. (A) Quantitative real-time PCR assay. RNA extracts of U87MG cells post-radiation and control cells were analyzed by quantitative RT-PCR to confirm the expression of miR-181a. Data are expressed as percentage of U6 content. MiR-181a expression was 2.12-fold decreased in response to radiation $(\mathrm{P}<0.05)$. (B) Western blot analysis of the predicted target of miR181a in U87MG cells. Twenty-four, 48 and $72 \mathrm{~h}$ post-transfection of miR181a mimic or control mimic, whole cell lysates were prepared and samples subjected to Western blot analysis for Bcl-2, and B-actin was used as a loading control. (C) Quantified Western blot analysis of miR-521 predicted $\mathrm{Bcl}-2$ as target. The relative $\mathrm{Bcl}-2$ protein levels were estimated using densitometry by Image $\mathbf{J}$ and normalized to $\beta$-actin. Bcl-2 was significantly decreased $72 \mathrm{~h}$ after transfection $\left({ }^{*} \mathrm{P}<0.05\right)$.

some variability, miR-181a showed a 2.12-fold decrease which matched the result of the microarray (Fig. 2A).
Exogenous miR-181a expression down-regulates Bcl-2 and modulates radiosensitivity. TargetScanHuman 5.1 (http://www. targetscan.org) was used for prediction of miR-181a target genes. The sequence alignment of human miR-181a with different species of Bcl-2 3'UTR is conserved, which indicates that Bcl-2 is one of the potential targets of miR-181a. Since $\mathrm{Bcl}-2$ is a pro-survival protein, we hypothesized that miR-181a might sensitize U87MG cells to radiotherapy by repressing the $\mathrm{Bcl}-2$ protein expression. In miR-181a mimic-treated U87MG cells, Bcl-2 was significantly decreased $72 \mathrm{~h}$ after transfection (Fig. 2B and C), suggesting that up-regulation of miR-181a resulted in decrease of Bcl-2 protein.

MiR-181a was down-regulated in response to radiation, so we hypothesized that overexpression of miR-181a could alter radiation response. First, we evaluated the potential effect of miR-181a overexpression on U87MG cell growth and survival without irradiation in vitro. The results from cell proliferation assays and soft agar colony formation assays showed that there were no significant decrease in the cell growth rate (Fig. 3A) and the potential of anchorage-independent growth (Fig. 4) in miR-181a overexpressed U87MG compared with the cells transfected with control mimic. Then in the other group, cells also transfected with miR-181a mimic or control mimic were exposed to radiation. The results from MTT assays showed that after $18.8 \mathrm{~Gy}$ irradiation, the cell growth rate was significantly reduced in miR-181a overexpressed cells (Fig. 3B). Colony formation assays demonstrated similar radiation effects (Fig. 4). Control U87MG presented radioresistance at $2 \mathrm{~Gy}$ irradiation. Conversely, U87MG transfected with the miR-181a mimic had significant lower colony formation ability after 2 Gy irradiation. When cells were subjected to a higher irradiation dose (5 Gy), the miR-181a mimic-treated U87MG was significantly more sensitive to radiation compared to the control. These results suggest that miR-181a sensitizes U87MG cells to radiotherapy.

\section{Discussion}

Radiation therapy is routinely prescribed for high-grade malignant gliomas. However, the occurrence of radioresistance often affects the therapeutic efficacy and the disease recur after initial treatment in most cases. The gene expression pattern and molecular response of malignant glioma cells to radiation inhibit the undergoing apoptosis and contribute to the development of radioresistance (21). But the genetic response necessary for tumors to survive radiationinduced injury remains largely unknown.

MiRNAs regulate protein expression by cleaving or repressing the translation of target mRNAs. Evidence from 
A



B

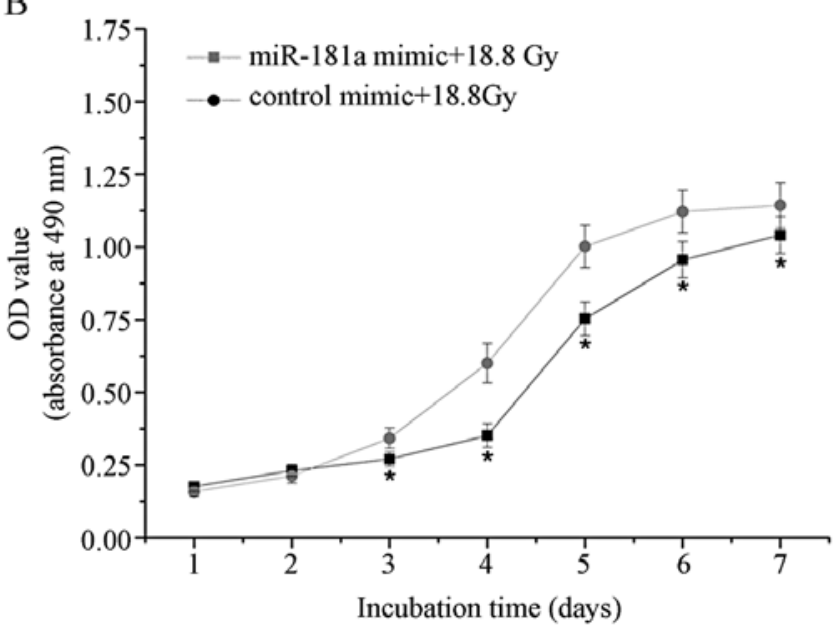

Figure 3. Cell growth evaluated by MTT assay. U87MG cells transfected with miR-181a mimic or control mimic were divided into two groups. (A) One group received no radiation treatment. At $490 \mathrm{~nm}$, the results of viability showed no significant differences between the miR-181a up-regulated cells and the control. (B) After exposure to 18.8 Gy X-ray, the viability of miR-181a up-regulated U87MG cells was significantly decreased and the highest inhibition rate was $41.6 \%$ on day 4 . The experiment was performed in triplicate (a paired Student's t-test indicated statistical significance between the two curves, ${ }^{*} \mathrm{P}<0.05$ ).

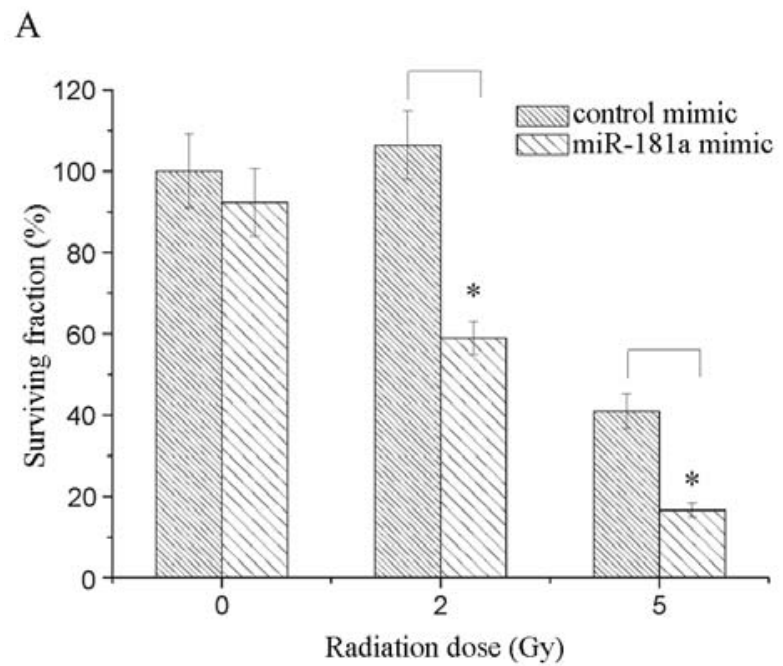

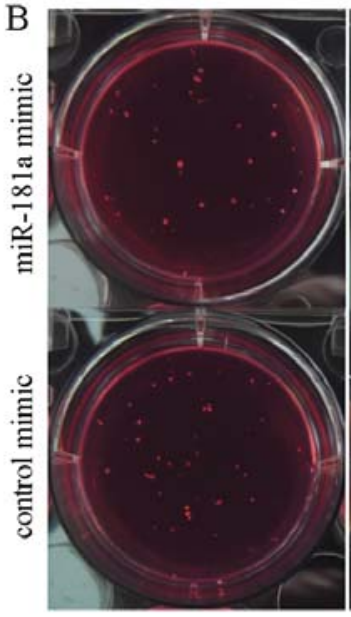

0 Gy

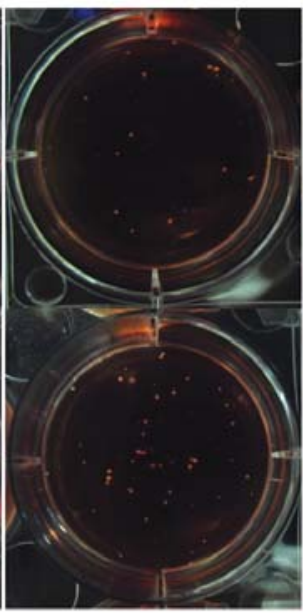

2 Gy

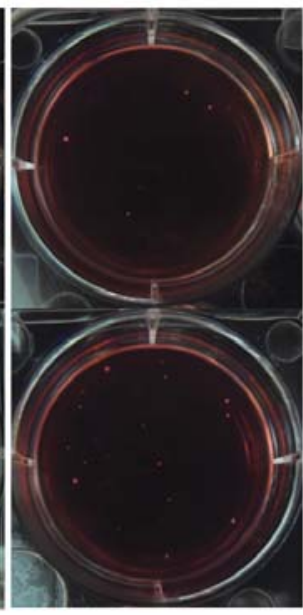

5 Gy

Figure 4. Anchorage-independent growth assessed by colony formation in soft agar. Ectopic miR-181a expression reduced the anchorage-independent growth of radiation treated U87MG cells. U87MG cells were transfected with miR-181a mimic or control mimic. Following irradiation (0, 2 and 5 Gy), cells were incubated for 3 weeks. (A) Colonies containing $\geq 50$ cells were scored. Data are expressed as the survival fractions of cells vs. the radiation dose. Survival fraction of non-irradiated U87MG cells transfected with control mimic was defined as $100 \%$. Colony formation assays showed that miR-181a overexpressed cells had significant lower colony formation ability after 2 and 5 Gy irradiation $\left({ }^{*} \mathrm{P}<0.05\right)$. The experiments were repeated 3 times. (B) The photograph is a representative example.

recent studies shows the relationship between miRNAs and the radioresistance. So the miRNAs are potential targets of our interest, and biomarkers of outcome. However, little is known about how they affect the response of a tumor to this cytotoxic treatment.

To determine whether miRNAs were involved in the cellular response to radiotherapy in malignant glioma, miRNA microarrays were used to compare the relative levels of cellular miRNAs before and after irradiation. We selected the GBM cell line U87MG which is a relatively radioresistant cell line, in which apoptosis is not triggered until $72 \mathrm{~h}$ (1). First, radiosensitivity was tested to determine the radiation dose. Then irradiation was delivered with conventional fractionation, simulating the clinical radiation treatment plan delivered to patients with malignant glioma (16) and one beam direction only was used rather than the typical five in clinical practice. Four hours after the whole procedure of the irradiation, total RNA was extracted and subjected to the microarray assay. Most miRNAs did not show strong response in expression except 13 miRNAs were up-regulated and 8 down-regulated. These findings suggest that the miRNAs are involved in the cellular response of U87MG to radiation insult. Of the 21 miRNAs with changed expression after irradiation, several have been reported to be associated with radiosensitivity. Let-7g level is increased in response to radiation in human lung cancer cell line A549 and its overexpression causes 
significant radioprotection in the cells (6). MiR-521 level is significantly decreased in prostate cancer cell lines after irradiation. Ectopic miR-521 expression represses its predicted target protein CSA (Cockayne syndrome protein A) and enhances the radiosensitivity of the prostate cancer cells (7). Expression changes respond to radiation of miR-22 (22), 379 (7), 548 (8) also have been reported recently. These findings prompted us to test the hypothesis that altering one or several miRNAs could be an efficacious method to alter the cellular radiosensitivy in malignant glioma.

QRT-PCR was used to confirm the expression changes of several radiation-responsive miRNAs. The downregulation of miR-181a was confirmed as a $>2$-fold change. Then the targets of this radiation-responsive miRNA were predicted using TargetScanHuman 5.1. More than 1,000 potential target genes were predicted, and we chose several proteins having functions related to apoptosis, cell cycle control, and DNA damage and repair. Bcl-2 was one of the candidates.

Bcl-2 is an important member of a protein family composed of regulators of programmed cell death in both normal and abnormal cells. It is a pro-survival protein that regulates apoptosis by preventing the release of pro-apoptotic factors from mitochondria (e.g. cytochrome c) and subsequent activation of a caspase cascade $(14,23,24)$. In vitro, exposure of cancer cells to radiation resulted in the up-regulation of $\mathrm{Bcl}-2$, indicating these cells were attempting to adapt to the harmful environment $(21,25,26)$. Bcl-2 expression can result in the development of radiation-resistant cancers. It has been reported that down-regulation of PTEN results in upregulation of $\mathrm{Akt}$ and $\mathrm{Bcl}-2$, thus illustrating the possible cross-talk between the Bcl-2 and PI3K/Akt pathway (27).

These findings accompanied by the target prediction outcome above made us believe that there might be a vital molecular link between miR-181a and Bcl-2. Compared with control miRNA mimic, up-regulation of miR-181a by transfection with the miR-181a mimic resulted in the downregulation of $\mathrm{Bcl}-2$ protein, concurrent with the enhanced effect of radiation treatment on malignant glioma cells U87MG. These data suggested that miR-181a modulated radiosensitivity by targeting $\mathrm{Bcl}-2$ in $\mathrm{U} 87 \mathrm{MG}$.

Although miR-181a is a modulator of radiosensitivity in glioma, the understanding of miR-181a is still fragmented. Bcl-2 may be just one of its targets. It is very possible that miR-181a is also involved in other molecular networks which disrupt the pro-survival signaling pathways.

In summary, our findings have demonstrated that a set of human miRNAs show significant expression changes in response to radiation, among which miR-181a is downregulated. It modulates the expression levels of Bcl-2 and plays an important role in modulating radiosensitivity of the malignant cell line U87MG. Thus miR-181a can be a potential target for enhancing the effect of radiotherapy on malignant glioma.

\section{Acknowledgements}

We would like to thank Chengchun Fu for helpful suggestions in the radiation treatments and $\mathrm{Nan} \mathrm{Xu}$ for technical assistance.

\section{References}

1. Brondani Da Rocha A, Regner A, Grivicich I, Pretto Schunemann D, Diel C, Kovaleski G, Brunetto De Farias C, Mondadori E, Almeida L, Braga Filho A and Schwartsmann G: Radioresistance is associated to increased Hsp70 content in human glioblastoma cell lines. Int J Oncol 25: 777-785, 2004.

2. Osada $\mathrm{H}$ and Takahashi T: MicroRNAs in biological processes and carcinogenesis. Carcinogenesis 28: 2-12, 2007.

3. He L, He X, Lim LP, de Stanchina E, Xuan Z, Liang Y, Xue W, Zender L, Magnus J, Ridzon D, Jackson AL, Linsley PS, Chen C, Lowe SW, Cleary MA and Hannon GJ: A microRNA component of the p53 tumour suppressor network. Nature 447: 1130-1134, 2007.

4. Ilnytskyy Y, Zemp FJ, Koturbash I and Kovalchuk O: Altered microRNA expression patterns in irradiated hematopoietic tissues suggest a sex-specific protective mechanism. Biochem Biophys Res Commun 377: 41-45, 2008.

5. Kato M, Paranjape T, Ullrich R, Nallur S, Gillespie E, Keane K, Esquela-Kerscher A, Weidhaas JB and Slack FJ: The mir-34 microRNA is required for the DNA damage response in vivo in C. elegans and in vitro in human breast cancer cells. Oncogene 28: 2419-2424, 2009.

6. Weidhaas JB, Babar I, Nallur SM, Trang P, Roush S, Boehm M, Gillespie E and Slack FJ: MicroRNAs as potential agents to alter resistance to cytotoxic anticancer therapy. Cancer Res 67: 11111-11116, 2007.

7. Josson S, Sung SY, Lao K, Chung LW and Johnstone PA: Radiation modulation of microRNA in prostate cancer cell lines. Prostate 68: 1599-1606, 2008.

8. Maes OC, An J, Sarojini H, Wu H and Wang E: Changes in MicroRNA expression patterns in human fibroblasts after lowLET radiation. J Cell Biochem 105: 824-834, 2008.

9. Lee JU, Hosotani R, Wada M, Doi R, Kosiba T, Fujimoto K, Miyamoto Y, Tsuji S, Nakajima S, Nishimura Y and Imamura M: Role of Bcl-2 family proteins (Bax, Bcl-2 and Bcl-X) on cellular susceptibility to radiation in pancreatic cancer cells. Eur J Cancer 35: 1374-1380, 1999

10. Belka C and Budach W: Anti-apoptotic Bcl-2 proteins: structure, function and relevance for radiation biology. Int $\mathrm{J}$ Radiat Biol 78: 643-658, 2002.

11. Wang S, Yang D and Lippman ME: Targeting Bcl-2 and Bcl-XL with nonpeptidic small-molecule antagonists. Semin Oncol 30: 133-142, 2003.

12. Reed JC: Drug insight: cancer therapy strategies based on restoration of endogenous cell death mechanisms. Nat Clin Pract Oncol 3: 388-398, 2006.

13. Xia L, Zhang D, Du R, Pan Y, Zhao L, Sun S, Hong L, Liu J and Fan D: miR-15b and miR-16 modulate multidrug resistance by targeting BCL2 in human gastric cancer cells. Int J Cancer 123: 372-379, 2008.

14. Lucken-Ardjomande S and Martinou JC: Regulation of Bcl-2 proteins and of the permeability of the outer mitochondrial membrane. C R Biol 328: 616-631, 2005.

15. An J, Chervin AS, Nie A, Ducoff HS and Huang Z: Overcoming the radioresistance of prostate cancer cells with a novel Bcl-2 inhibitor. Oncogene 26: 652-661, 2007.

16. Chan JL, Lee SW, Fraass BA, Normolle DP, Greenberg HS, Junck LR, Gebarski SS and Sandler HM: Survival and failure patterns of high-grade gliomas after three-dimensional conformal radiotherapy. J Clin Oncol 20: 1635-1642, 2002.

17. Castoldi M, Schmidt S, Benes V, Noerholm M, Kulozik AE, Hentze MW and Muckenthaler MU: A sensitive array for microRNA expression profiling (miChip) based on locked nucleic acids (LNA). RNA 12: 913-920, 2006.

18. Chen C, Ridzon DA, Broomer AJ, Zhou Z, Lee DH, Nguyen JT, Barbisin M, Xu NL, Mahuvakar VR, Andersen MR, Lao KQ, Livak KJ and Guegler KJ: Real-time quantification of microRNAs by stem-loop RT-PCR. Nucleic Acids Res 33: e179, 2005.

19. Livak KJ and Schmittgen TD: Analysis of relative gene expression data using real-time quantitative PCR and the 2(-Delta Delta C(T)) method. Methods 25: 402-408, 2001.

20. Zeng Y, Zhang X, Huang Z, Cheng L, Yao S, Qin D, Chen X, Tang Q, Lv Z, Zhang L and Lu C: Intracellular Tat of human immunodeficiency virus type 1 activates lytic cycle replication of Kaposi's sarcoma-associated herpesvirus: role of JAK/STAT signaling. J Virol 81: 2401-2417, 2007.

21. Guo WF, Lin RX, Huang J, Zhou Z, Yang J, Guo GZ and Wang SQ: Identification of differentially expressed genes contributing to radioresistance in lung cancer cells using microarray analysis. Radiat Res 164: 27-35, 2005. 
22. Shin S, Cha HJ, Lee EM, Lee SJ, Seo SK, Jin HO, Park IC, Jin YW and An S: Alteration of miRNA profiles by ionizing radiation in A549 human non-small cell lung cancer cells. Int J Oncol 35: 81-86, 2009.

23. Reed JC: Bcl-2 and the regulation of programmed cell death. J Cell Biol 124: 1-6, 1994.

24. Yang E and Korsmeyer SJ: Molecular thanatopsis: a discourse on the BCL2 family and cell death. Blood 88: 386-401, 1996.

25. Park SH, Cho HN, Lee SJ, Kim TH, Lee Y, Park YM, Lee YJ, Cho CK, Yoo SY and Lee YS: Hsp25-induced radioresistance is associated with reduction of death by apoptosis: involvement of Bcl2 and the cell cycle. Radiat Res 154: 421-428, 2000.
26. Wang Q, Gao F, May WS, Zhang Y, Flagg T and Deng X: Bcl2 negatively regulates DNA double-strand-break repair through a nonhomologous end-joining pathway. Mol Cell 29: 488-498, 2008.

27. Rosser CJ, Gaar M and Porvasnik S: Molecular fingerprinting of radiation resistant tumors: can we apprehend and rehabilitate the suspects? BMC Cancer 9: 225, 2009. 